\title{
CAPÍTULO 15: ENSINO REMOTO, ACESSO ÀS TECNOLOGIAS DE INFORMAÇÃO E COMUNICAÇÃO: A SITUAÇÃO DE ESCOLAS E ALUNOS NO BRASIL
}

\section{CAPÍTULO 15: ENSEÑANZA REMOTA, ACCESO AS TECNOLOGÍAS DE INFORMACION Y COMUNICACIÓN: LA SITUACIÓN DE ESCUELAS Y ESTUDIANTES EN BRASIL}

\section{CHAPTER 15: REMOTE EDUCATION, ACCESS TO INFORMATION AND COMMUNICATION TECHNOLOGIES: THE SITUATION OF SCHOOLS AND STUDENTS IN BRAZIL}

\begin{abstract}
Breno Trajano de Almeida ${ }^{1}$; Leonardo Rafael Medeiros ${ }^{2}$; Louize Gabriela Silva de Souza ${ }^{3}$
\end{abstract}
DOI: https://doi.org/10.31692/978-65-88970-05-8.205-210

\section{INTRODUÇÃO}

Em 31 de dezembro de 2019, autoridades chinesas alertaram a Organização Mundial da Saúde (OMS) sobre diversos casos de pneumonia de origem desconhecida na cidade de Wuhan. Setenta dias depois, em 11 de março, a OMS declarou que o mundo estava vivendo uma pandemia de COVID-19, causada por um novo coronavírus, o Sars-CoV-2 (UNA-SUS, ONU News, mar. 2020). Em sequência, tudo mudou. A insegurança frente ao invisível desconhecido alterou as rotinas, o trabalho, a convivência, o contato. E as escolas precisaram ser fechadas, como condição para a não proliferação do vírus. Mas o que fazer com os alunos? Com o ano letivo? Quais as condições para chegar até cada criança, cada jovem, cada adulto matriculado numa das 180.610 escolas de educação básica (Inep, 2019) do Brasil, já tão marcado por desigualdades sociais e estruturais? Diante desse contexto, se intenta apresentar aqui, um panorama sucinto das condições de acesso e utilização das tecnologias de informação e comunicação e que impacta nas possibilidades de aulas remotas na educação básica.

\footnotetext{
${ }^{1}$ Professor do Instituto Federal de Educação, Ciência e Tecnologia do Rio Grande do Norte (IFRN) - Campus Ipanguaçu, breno.almeida@ifrn.edu.br

${ }^{2}$ Professor do Instituto Federal de Educação, Ciência e Tecnologia do Rio Grande do Norte (IFRN) - Campus Ipanguaçu, leonardo.rafael@ifrn.edu.br

${ }^{3}$ Professora do Instituto Federal de Educação, Ciência e Tecnologia do Rio Grande đo Norte (IFRN) - Campus Ipanguaçu, louize.gabriela@ifrn.edu.br
} 


\section{FUNDAMENTAÇÃO TEÓRICA}

Muito embora os efeitos da COVID-19 tenham afetado as pessoas acometidas pela doença, seus desdobramentos impactaram sobremaneira todas as esferas da sociedade. $\mathrm{Na}$ educação, até 25 de março de 2020 cerca de 1,5 bilhão de estudantes tiveram "aulas suspensas ou reconfiguradas ao redor do mundo" (UNESCO, 2020a), representando mais de 90\% de todos os estudantes do planeta. Ainda segundo a UNESCO, que faz acompanhamento diário, cerca de 580 milhões de estudantes, no mundo, permanecem sem aulas presenciais, em todos os níveis.

Inicialmente, as aulas remotas pela internet, por meio de ambientes virtuais de aprendizagem - AVA (ou LMS, na sigla em inglês), foram recomendadas pelas Organizações de Saúde para o Ensino Superior. Na Educação Básica, com aulas suspensas desde o início de março, a recomendação de que aulas não presencias pudessem contar como carga horária (no mínimo, 800 h anuais, obrigatórias ${ }^{1}$ ) foi sinalizada pelo Conselho Nacional de Educação CNE, em 28 de abril, por meio do Parecer CNE/CP n 05/2020, possibilitando que as escolas, públicas e privadas, adotassem estratégias de ensino remoto para os alunos, da educação infantil ao ensino médio.

Ao apontar "Estratégias de ensino a distância em resposta ao fechamento das escolas devido a COVID-19”, a UNESCO (abr. 2020b) levanta dois pontos, entre outros, que se julga fundamentais na implementação de aulas remotas: i) apoio aos "grupos desfavorecidos - alunos sem acesso a aparelhos de rádio, TV ou digitais conectados e estudantes com alguma deficiência"; ii) incluir "plataformas de aprendizagem administradas por governos, órgãos públicos ou universidades" como recursos tecnológicos para aprendizagem a distância e "apoio à oferta de lições, hospedagem e gerenciamento de materiais didáticos, e suporte a comunicação entre professores e estudantes". Nesse contexto, e em meio à declaração de que as desigualdades de acesso à internet "vão afetar a escola, mas isso aí já é para um outro departamento, de assistência. Não tenho como resolver isso", do Ministro da Educação ${ }^{2}$ é preciso saber como se configuram o acesso e o uso de tecnologias de informação e comunicação no universo da educação básica no Brasil.

\footnotetext{
${ }^{1}$ Lei 939/1996, Art. 24, I. http://www.planalto.gov.br/ccivil 03/leis/L9394compilado.htm Acesso: out/2020.

${ }^{2}$ Entrevista ao Estadão, em 24/09/2020. https://educacao.estadao.com.br/noticias/geral,voltas-as-aulas-no-pais acesso-a-web-nao-sao-temas-do-mec-diz-ministro,70003450120. Acesso: out/2020.

[206]
} 


\section{METODOLOGIA}

Essa pesquisa, de natureza quantitativa e qualitativa, utilizou dados disponíveis em órgãos oficiais, buscando percentuais de equipamentos e de acesso à tecnologias de informação e comunicação, num comparativo entre as regiões brasileiras. Esses dados são relevantes para aferir o alcance das aulas remotas, consequência dos efeitos da pandemia por COVID-19.

\section{RESULTADOS E DISCUSSÃO}

Para que aulas remotas aconteçam é necessário ter rede de internet, celular, televisor e telefone, além de outros elementos que garantam o acesso dos estudantes às atividades. Nesse sentido, a própria distribuição das matrículas da educação básica, considerando rede e localização das escolas, nas regiões geográficas e a disponibilidade de recursos tecnológicos nas instituições de ensino permitem inferir onde estão os gargalos. Segundo o Censo Escolar 2019 (Inep, 2019), são 47.874.246 matrículas (42.545.428 urbanas e 5.328.818 rurais), com a seguinte distribuição por esfera administrativa e grandes regiões:

Tabela 1: Matrículas Educação Básica

\begin{tabular}{l|r|r|r|r|r|r|r|r}
\hline REDE & \multicolumn{2}{|c|}{ FEDERAL } & \multicolumn{2}{c|}{ ESTADUAL } & \multicolumn{2}{c|}{ MUNICIPAL } & \multicolumn{2}{c}{ PRIVADA } \\
\hline ÁREA & Urbana & \multicolumn{1}{c}{ Rural } & Urbana & \multicolumn{1}{c}{ Rural } & Urbana & \multicolumn{1}{c}{ Rural } & \multicolumn{1}{c}{ Urbana } & \multicolumn{1}{c}{ Rural } \\
\hline Norte & 44.246 & 3.545 & 1.512 .093 & 208.328 & 1.765 .682 & 930.446 & 452.957 & 7.366 \\
\hline Nordeste & 113.064 & 17.561 & 2.884 .167 & 229.217 & 5.589 .650 & 2.629 .883 & 2.459 .040 & 45.894 \\
\hline Sudeste & 103.331 & 15.002 & 6.358 .254 & 156.176 & 7.446 .611 & 435.869 & 4.296 .755 & 27.028 \\
\hline Sul & 66.446 & 6.011 & 2.271 .036 & 128.138 & 2.592 .216 & 243.913 & 1.164 .652 & 4.006 \\
\hline C.Oeste & 28.744 & 7.857 & 1.464 .077 & 95.547 & 1.260 .934 & 132.417 & 672.473 & 4.614 \\
\hline Total & \multicolumn{6}{|c|}{405.807} & \multicolumn{6}{c|}{15.307 .033} & \multicolumn{2}{c}{23.027 .621} & \multicolumn{2}{c}{9.134 .785} \\
\hline
\end{tabular}

Fonte: Inep. Censo Escolar 2019

Essas matrículas estão distribuídas em 180.610 escolas $^{3}$ com grandes variações no acesso e disponibilização de recursos tecnológicos:

Tabela 2: Tecnologias de Comunicação e Educação nas escolas de Ed. Básica (\%)

\begin{tabular}{|c|c|c|c|c|c|c|c|c|}
\hline REDE & \multicolumn{2}{|c|}{ FEDERAL } & \multicolumn{2}{|c|}{ ESTADUAL } & \multicolumn{2}{|c|}{ MUNICIPAL } & \multicolumn{2}{|c|}{ PRIVADA } \\
\hline ETAPA & E. F & E.M & E. F & E.M & E. F & E.M & E. F & E.M \\
\hline NÚMERO DE ESCOLAS & 47 & 586 & 22.403 & 19.678 & 78.794 & 188 & 24.922 & 8.408 \\
\hline Comput. de mesa para alunos & 89,4 & 93,3 & 75,4 & 78,7 & 34,3 & 61,7 & 59,0 & 75,8 \\
\hline Comput. portátil para alunos & 51,1 & 44,9 & 33,5 & 38,5 & 20,4 & 35,1 & 41,2 & 49,2 \\
\hline Tablet para alunos & 34,0 & 25,8 & 13,4 & 15,4 & 5,7 & 7,4 & 24,5 & 30,7 \\
\hline Internet & 100 & 99,8 & 86,7 & 90,2 & 61,5 & 86,7 & 93,1 & 93,6 \\
\hline Internet para alunos & 83,0 & 96,8 & 57,2 & 61,0 & 21,2 & 44,7 & 44,0 & 63,2 \\
\hline Internet p/ens. e aprendizagem & 80,9 & 86,2 & 64,0 & 65,8 & 29,6 & 52,7 & 57,4 & 72,3 \\
\hline
\end{tabular}

Fonte: Deed/Inep com base nos dados do Censo Escolar 2019

\footnotetext{
${ }^{3} \mathrm{O}$ mesmo estabelecimento pode oferecer mais de uma etapa de ensino e aparecer mais de uma vez no Censo.
} 
Embora a rede municipal seja a maior, em número de matrículas e escolas, é a que menos tem recursos tecnológicos em todos os itens destacados. Esses números sinalizam que o uso para ensino e aprendizagem ainda é pouco utilizado, tanto por alunos quanto por professores, seja por indisponibilidade dos equipamentos e/ou cobertura da internet, seja por falta de formação para o uso dessas tecnologias. Além disso, mais de $10 \%$ dos municípios brasileiros não têm cobertura de internet e 45,9 milhões de brasileiros não têm acesso à internet, o que representa $25,3 \%$ da população com 10 anos ou mais, de acordo com a Pesquisa por Amostra de Domicílio Contínua - PNAD, 2018 (IBGE, 2019).

Outros dados que evidenciam as dificuldades para um efetivo, mesmo que temporário, ensino remoto, estão demostrados nas lacunas de utilização de internet, existência de TV e telefone no domicílio, equipamento de acesso à internet e idade dos usuários:

Gráfico 1: Percentual de utilização da Internet por Grandes Regiões

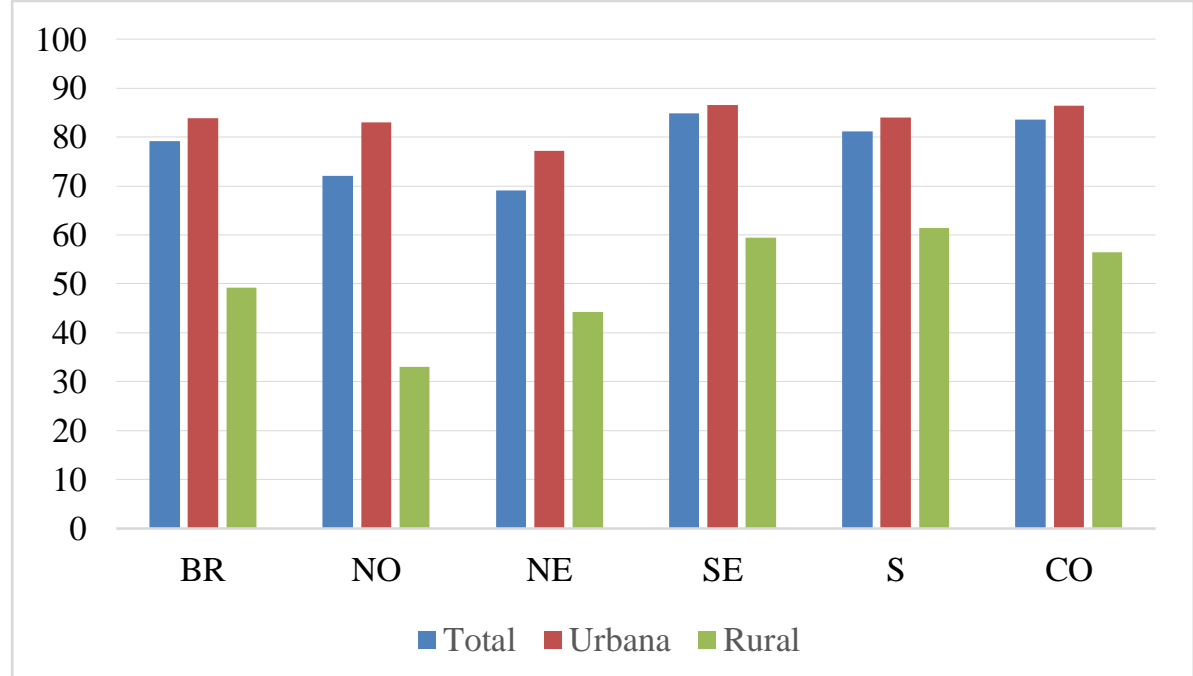

Fonte: IBGE. Diretoria de Pesquisa. Coordenação de Trabalho e Rendimento. Pesquisa por Amostra de Domicílio Contínua, 2018.

Gráfico 2: Percentual de domicílios com TV, telefone fixo e celular

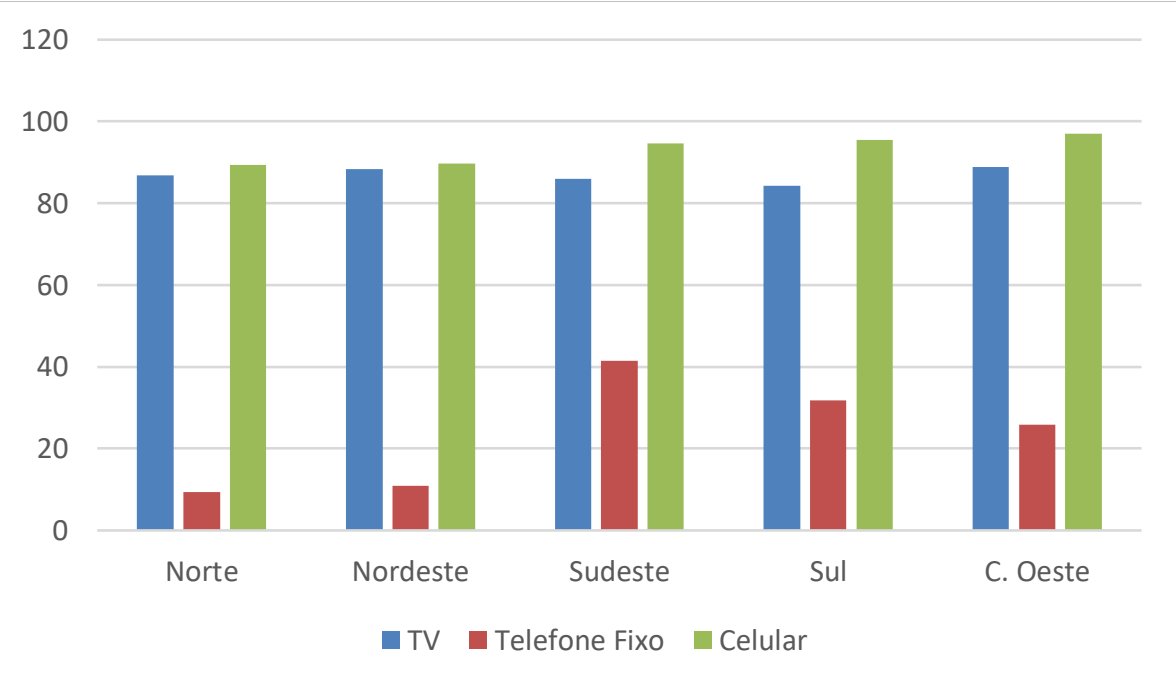

Fonte: IBGE. Diretoria de Pesquisa. Coordenação de Trabalho e Rendimento. Pesquisa por Amostra de 


\section{Domicílio Contínua, 2018.}

Gráfico 3: Percentual de pessoas por equipamento de acesso à internet.

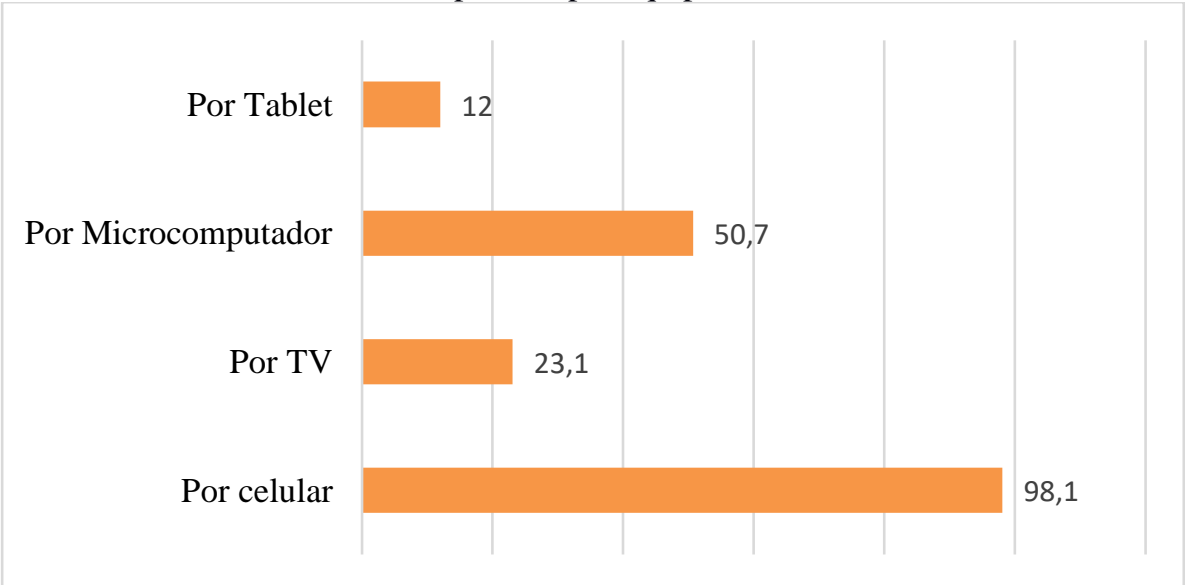

Fonte: IBGE. Diretoria de Pesquisa. Coordenação de Trabalho e Rendimento. Pesquisa por Amostra de Domicílio Contínua, 2018.

Gráfico 4: Percentual de pessoas, por idade, que mais acessam internet.

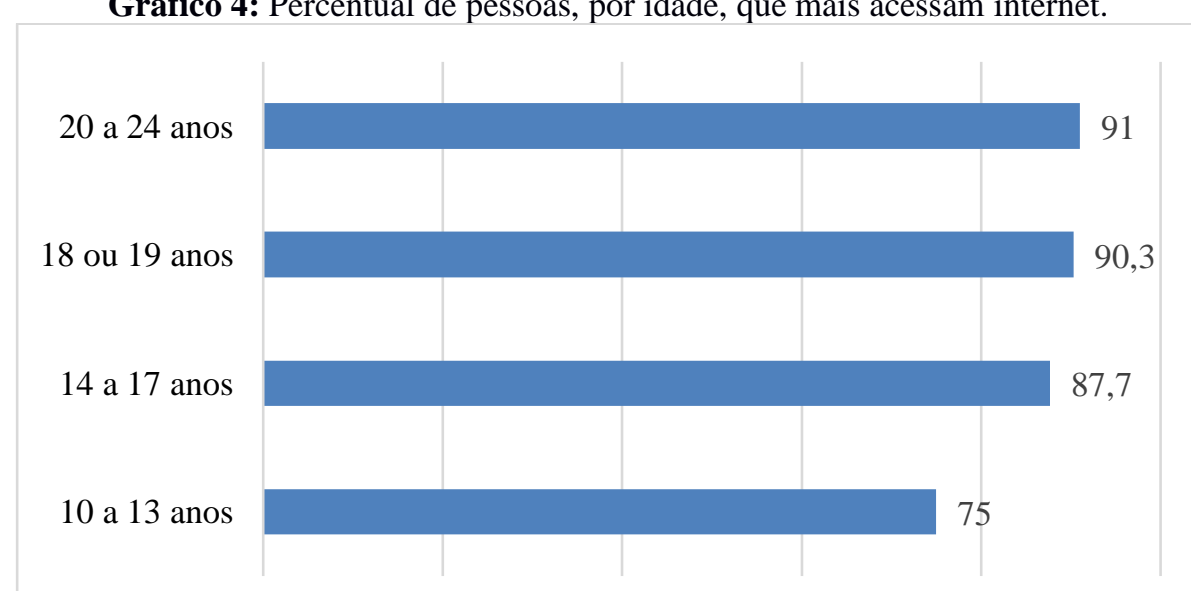

Fonte: IBGE. Diretoria de Pesquisa. Coordenação de Trabalho e Rendimento. Pesquisa por Amostra de Domicílio Contínua, 2018.

\section{CONCLUSÕES}

O pouco tempo para adaptações e para aprender sobre aulas pela internet, TV, mensagens ou redes sociais, aliado às condições das famílias $(52,6 \%$ dos brasileiros com 25 anos, ou mais, não possuem o mínimo de estudo esperado) em acompanhar as atividades dos filhos, estressa professores e gestores na busca de soluções e cria uma incógnita sobre o processo educacional que, possivelmente, não retornará tão cedo aos moldes anteriores, se confirmados os prognósticos sobre vacinas e cuidados mais permanentes.

\section{REFERÊNCIAS}

IBGE: Pesquisa por Amostra de Domicílio Contínua, PNAD, 2018. Disponível em: 
https://www.ibge.gov.br/estatisticas/multidominio/condicoes-de-vida-desigualdade-epobreza/17270-pnad-continua.html?=\&t=o-que-e. Acesso: out.2020

Instituto Nacional de Estudos e Pesquisas Educacionais Anísio Teixeira. Sinopse Estatística da Educação Básica 2019. Brasília: Inep, 2020. Disponível em: http://portal.inep.gov.br/web/guest/sinopses-estatisticas-da-educacao-basica Acesso: out. 2020

ONU News. Disponível em: https://news.un.org/pt/story/2020/03/1706881 Acesso: out.2020

Sistema Universidade Aberta do SUS (UNA-SUS) Disponível em:

https://www.unasus.gov.br/noticia/organizacao-mundial-de-saude-declara-pandemia-decoronavirus. Acesso: out. 2020

UNESCO. Impacto do Covid-19 na educação. 2020a. Disponível em:

https://en.unesco.org/covid19/educationresponse. Acesso: out. 2020

. Nota informativa $\mathrm{n}^{\circ} 2.1 .2020 \mathrm{~b}$. Disponível em:

https://unesdoc.unesco.org/ark:/48223/pf0000373305_por. Acesso: out. 2020 